\title{
Hasse diagram as a green analytical metrics tool: ranking of methods for benzo[a]pyrene determination in sediments
}

\author{
Paulina Bigus $^{1} \cdot$ Stefan Tsakovski $^{2} \cdot$ Vasil Simeonov $^{2}$ - Jacek Namieśnik ${ }^{1}$. \\ Marek Tobiszewski ${ }^{1}$
}

Received: 28 January 2016/Revised: 29 February 2016 / Accepted: 7 March 2016 / Published online: 1 April 2016

(C) The Author(s) 2016. This article is published with open access at Springerlink.com

\begin{abstract}
This study presents an application of the Hasse diagram technique (HDT) as the assessment tool to select the most appropriate analytical procedures according to their greenness or the best analytical performance. The dataset consists of analytical procedures for benzo[ $a]$ pyrene determination in sediment samples, which were described by 11 variables concerning their greenness and analytical performance. Two analyses with the HDT were performed - the first one with metrological variables and the second one with "green" variables as input data. Both HDT analyses ranked different analytical procedures as the most valuable, suggesting that green analytical chemistry is not in accordance with metrology when benzo $[a]$ pyrene in sediment samples is determined. The HDT can be used as a good decision support tool to choose the proper analytical procedure concerning green analytical chemistry principles and analytical performance merits.
\end{abstract}

Keywords Multivariate statistics · Greenness assessment · Green chemistry $\cdot$ Benzo $[a]$ pyrene $\cdot$ Chemometrics

Electronic supplementary material The online version of this article (doi:10.1007/s00216-016-9473-4) contains supplementary material, which is available to authorized users.

Marek Tobiszewski

marektobiszewski@wp.pl

1 Department of Analytical Chemistry, Chemical Faculty, Gdańsk University of Technology (GUT), 11/12 G. Narutowicza St., 80-233 Gdańsk, Poland

2 Chair of Analytical Chemistry, Faculty of Pharmacy and Chemistry, University of Sofia "St. K1. Okhridski”, J. Bourchier Blvd. 1, 1164 Sofia, Bulgaria

\section{Introduction}

Polycyclic aromatic hydrocarbons (PAHs) include a wide class of hazardous organic compounds that consist of two or more benzene rings in linear, angular, or cluster arrangements [1]. Because of the short-term and long-term toxicity of PAHs and their persistence or ability to bioaccummulate in the environment, these compounds have become the focus of much attention in recent years [2]. Sixteen PAHs known as priority pollutants are on the lists established by agencies responsible for environmental resource management in Europe and the USA [3]. Some species of PAHs have been classified as either possible or probable human carcinogens and mutagens by experts from the International Agency for Research on Cancer [4]. PAHs are metabolized to dihydrodiols by hydrocarbon hydroxylases present in the liver. Benzo $[a]$ pyrene as a potent carcinogenic species has been chosen by certain agencies and organizations as an indicator of total PAHs [5]. The toxicities of other PAHs are often compared with the toxicity of benzo $[a]$ pyrene.

PAHs are ubiquitous organic micropollutants [6]. They are mainly formed during the incomplete combustion of natural and organic materials (e.g., coal, fossil fuels, tobacco, or smoked meat) [7, 8]. Distribution of PAH mixtures into the environment is also caused by industrial emissions (e.g., coal gasification, aluminum production, steel founding) or traffic exhausts. Atmospheric PAHs, as particulates or gases, can be deposited on the surface of water. Because of their high lipophilicity and low biodegradability, PAHs can be absorbed by particulate materials rich in organic matter and accumulate at the bottom of lakes or rivers [9]. Hazardous compounds present in sediments can be a potential danger to aquatic ecosystems or drinking water. Understanding of the impact of particular emission sources on the different compartments of the aquatic 
environment is crucial for proper risk assessment and risk management. For this purpose reliable analytical procedures are required, and many have been proposed.

The quantitative determination of PAHs in sediments is a challenging task because of certain differences in the polarity, water solubility, and volatility of these compounds, their low concentration in samples, and the potential for the presence of matrix interferences. Thus, several methods have been described for the determination of PAHs, applying different extraction, purification, and detection methods. Chromatographic techniques, mainly gas chromatography [10] and highperformance liquid chromatography [11] have usually been applied for the determination of PAHs in sediments. Chromatographic analyses require sample pretreatment. For complex matrices such as sediment samples, Soxhlet extraction or liquid-solid extraction are the methods recommended by the US Environmental Protection Agency and the US National Oceanic and Atmospheric Administration [12, 13]. However, these extraction methods are highly time-consuming and labor intensive [14]. Moreover, their introduction in routine analysis is related to the use of a large volume of toxic solvents, which in consequence can result in long exposure of laboratory personnel. Increase in the energy costs and the amount of waste constitutes another very important aspect in analytical processes. Considering all these disadvantages, the sample preparation has proven to be the most significant stage in the whole analytical procedure and the most polluting in most procedures applying traditional methods [15].

The main goal of green analytical chemistry [16], which emerged from green chemistry, is to reach a compromise between the quality of the results and improving the environmental friendliness of analytical methods [17]. Because of special analytical criteria that need to be considered, such as limits of detection (LODs), limits of quantitation, precision, and accuracy, in some cases it is impossible to meet certain green analytical chemistry requirements in analytical methods [18]. In this situation, some approaches to make analytical procedures greener should be included.

Different methods can be used for determination of a single analyte. There are a few assessment methods allowing the evaluation of the "greenness" of each analytical procedure; use of the National Environmental Methods Index (NEMI) is one of them [19]. The greenness of an analytical method is assessed by use of the greenness profile symbol, which is divided into four fields, each describing different aspects of the environmental impact of the method; that is, (1) persistence, bioaccumulation potential, and toxicity, (2) hazardousness, (3) corrosiveness, and (4) waste. The procedure is considered green and the pictogram field is filled green if the following requirements are met: none of the chemicals used during the procedure are listed on the persistence, bioaccumulation potential, and toxicity chemicals lists or is present on the $\mathrm{K}, \mathrm{F}$, $\mathrm{P}$, or $\mathrm{U}$ hazardous lists ( $\mathrm{F}$ list for nonspecific source waste, $\mathrm{K}$ list for source-specific waste, and $\mathrm{P}$ and $\mathrm{U}$ lists for discarded commercial chemicals), the $\mathrm{pH}$ of the sample is within the 2-12 range, and less than $50 \mathrm{~g}$ of waste is produced.

The second very important tool used for assessing the greenness of analytical procedures is the analytical EcoScale [20]. The result of analysis with Eco-Scale is expressed as a number lower than 100 . Penalty points which are allotted for the amount and type of chemical reagent, energy consumption, analyst occupational hazard, solid waste generated, and the way solid waste is treated (or not treated) are subtracted from the initial value of 100 .

Benzo $[a]$ pyrene was chosen as the representative of the group of PAHs to be analyzed in this study. The aim of the study is to obtain information about similarities or dissimilarities between analytical procedures applied to the determination of benzo $[a]$ pyrene in sediments by use of multivariate statistical techniques, which will be significant in selecting the "latent" factors determining the greenness of analytical procedures studied. We investigate the possibility of applying the Hasse diagram technique (HDT) as a metrics tool for green analytical chemistry taking into account the analytical performance of the analytical procedures. The HDT allows for partial ordering of multivariate sets. The results obtained from analysis of Eco-Scale and NEMI as methods for the assessment of analytical method greenness will be also discussed.

\section{Materials and methods}

\section{Input data for the analysis}

All the analytical procedures included in the HDT analysis were developed for the determination of benzo $[a]$ pyrene in sediment samples. The input data for the multivariate statistical analysis were collected from published articles. The publishers' databases, including American Chemical Society, Taylor and Francis Online database, Royal Society of Chemistry Journals online database, ScienceDirect, SpringerLink, and Wiley Online Library database, were searched for the appropriate analytical procedures. No time frame or any limitations concerning the analytical techniques were applied. The procedure was included only if all the required information could be extracted from the article; 
otherwise the analytical procedure was rejected from further analysis. The analytical procedures extracted for the statistical analyses are presented in Table 1.
All the procedures presented in Table 1 are described by the variables in such a way that 26 of the procedures were described by both metrological and greenness data and all 41

Table 1 Analytical procedures used as input data for multivariate analysis of National Environmental Methods Index (NEMI; in the form of the number of green fields) and Eco-Scale results

No. Analytical technique NEMI score Eco-Scale score Reference

1 Ultrasonic solid-liquid extraction-thin-layer chromatography-high-performance liquid chromatography-diode array detection/ultraviolet detection

2 Ultrasonic solid-liquid extraction-high-performance liquid chromatographyprogrammable fluorescence detection

3 Ultrasonic solid-liquid extraction-high-performance liquid chromatography-programmable fluorescence detection

4 Ultrasonic micellar extraction-high performance liquid chromatography-ultraviolet detection

Vortex-assisted extraction-dispersive liquid-liquid microextraction-high-performance liquid chromatography-fluorescence detection

6 Ultrasonic-assisted extraction-matrix solid-phase dispersion-high-performance liquid chromatography-ultraviolet detection

7 Focused ultrasonic solid-liquid extraction-high-performance liquid chromatographyfluorescence detection

8 Micro focused ultrasonic solid-liquid extraction-high-performance liquid chromatography-fluorescence detection

9 Miniaturized homogenous liquid-liquid extraction-high-performance liquid chromatographyfluorescence detection

10 Microwave-assisted extraction-solid-phase extraction-liquid chromatography-photodiode array detection-mass spectrometry

11 In situ microwave-assisted extraction-high-performance liquid chromatographyphotodiode array detection

12 Microwave-assisted extraction-high-performance liquid chromatography-ultraviolet detection

13 Microwave-assisted extraction-high performance liquid chromatography-fluorescence detection

14 Microwave-assisted extraction-gas chromatography-mass spectrometry

15 Microwave-assisted extraction-2-dimensional gas chromatography-time-of-flight mass spectrometry

16 Microwave-assisted extraction-gas chromatography-mass spectrometry

17 Soxhlet extraction-gas chromatography-mass spectrometry

18 Microwave-assisted extraction-gas chromatography-mass spectrometry

19 Soxhlet extraction-gas chromatography-mass spectrometry

20 Accelerated solvent extraction-supercritical fluid extraction-gas chromatography-mass spectrometry

21 Focused ultrasonic solid-liquid extraction-gas chromatography-mass spectrometry

22 Microwave-assisted solid-phase extraction-gas chromatography-mass spectrometry

23 Microwave-assisted headspace solid-phase microextraction-gas chromatography-tandem mass spectrometry

24 Microwave-assisted micellar solid-phase microextraction-gas chromatography-mass spectrometry

25 Pressurized hot water extraction-solid-phase microextraction-gas chromatographymass spectrometry

26 Pressurized liquid extraction-stir bar sorptive extraction-thermal desorption-gas chromatographytriple quadrupole mass spectrometry

27 Pressurized liquid extraction-solid-phase extraction-gas chromatography-mass spectrometry

28 Pressurized liquid extraction-gas chromatography-mass spectrometry

29 Pressurized liquid extraction-large-volume injection-gas chromatography-mass spectrometry

30 Programmed temperature vaporization-gas chromatography-mass spectrometry

31 Solid-phase extraction-gas chromatography-mass spectrometry/selected ion storage

32 Solid-phase extraction-gas chromatography-quadrupole ion trap mass spectrometry

33 Solid-liquid extraction-gas chromatography-tandem mass spectrometry-pseudo multiple reaction monitoring

34 Ultrasonic solid-liquid extraction-solid-phase extraction-gas chromatography-mass spectrometry

35 Ultrasonic solid-liquid extraction-gas chromatography-mass spectrometry

36 Ultrasonic solid-liquid extraction-gas chromatography-mass spectrometry

37 Ultrasonic solid-liquid extraction-gas chromatography-mass spectrometry

38 Ultrasonic solid-liquid extraction-stir bar sorptive extraction-thermal desorption-gas chromatography-mass spectrometry

39 Ultrasonic solid-liquid extraction-gas chromatography-electron ionization tandem mass spectrometry 2

40 Ultrasonic solid-liquid extraction-thin-layer chromatography-gas chromatographyion trap mass spectrometry

41 Online dynamic microwave-assisted extraction-solid-phase extraction-gas chromatography-mass spectrometry

72

51

62

77

81

89

86

83

51

59

81

78

61

60

56

53

63

74

68

83

65

75

92

93

90

77

68

69

86

60

84

81

90

68

62

53

53
74
55

55

60

60

67
[21]

[36]

[37]

[38]

[43] 
procedures were characterized by greenness variables only. The variables included in the chemometric analyses are presented and explained in Table 2. Completeness of data availability was the requirement to include the procedure in the chemometric analyses.

For detailed information on the input data, see the electronic supplementary material.

\section{Principal component analysis}

Principal component analysis is a well-known method widely applied in analytical chemistry, so it will not be described. All principal component analysis calculations were performed in PLS Toolbox for MATLAB.

\section{Hasse diagram}

The HDT is applied to visualize relations of partial order between objects (in this case analytical procedures) described by a certain number of variables. The HDT is well described in the literature [54-56], and only a brief description will be given here.

In the HDT the ranking of analytical procedures is done with respect to variables (i.e., metrological or environmental), which is called the "information basis" (IB). The processed data matrix $\mathbf{Q}(N \times R)$ contains $N$ procedures and $R$ variables. Entry $\mathrm{y}_{i r}$ of $\mathbf{Q}$ is the numerical value of the $r$ th variable for the $i$ th procedure. Two objects $s$ and $t$ are comparable if

$s \leq t \Leftrightarrow y(s) \leq y(t)$,

$y(s) \leq y(t) \Leftrightarrow y_{r}(s) \leq y_{r}(t)$ for all $y_{r} \in \mathrm{IB}$.

If there is at least one $y_{r}$ for which $y_{r}(s)>y_{r}(t)$, then the objects $\mathrm{s}$ and $\mathrm{t}$ are incomparable. A partially ordered set can be easily developed by a covering relation matrix which collects relations between each pair of procedures.

The relations stored in the covering relation matrix can be visualized by a Hasse diagram. For construction of the Hasse diagram, a uniform orientation of the variables should be secured; that is, high variable values correspond to "bad" procedures and low values correspond to "good" procedures [57]. In the present study the procedures near the upper part of the Hasse diagram indicate procedures that are characterized by poorer analytical (Fig. 3) or environmental (Fig. 2) performances.

The objects in a Hasse diagram that are not covered by other objects are called "maximal objects." Objects which do not cover other objects are called "minimal objects." In some diagrams there are also isolated objects which can be considered as maximal and minimal objects at the same time (objects 24 and 29 in Fig. 2). A chain is a set of comparable objects; therefore levels can be defined as the longest chain within the diagram. An antichain is a set of mutually incomparable objects, located at one and the same level. The height of the diagram is the longest chain, and the longest antichain is its width.

The number of incomparable elements in the Hasse diagram may obviously constitute a limitation in the attempt to rank the objects (analytical procedures) according to their variables. To a certain extent this problem can be remedied through the application of the so-called linear extensions of the partial order ranking. A linear extension is a total order, where all covering relations of the partially ordered set are reproduced [58]. Because of the incomparable elements in the partial order ranking, a number of possible linear extensions correspond to one partial order. However, the number of linear extensions goes with $N$ ! for a partially ordered set with $N$ objects, and only for cases with a relatively low number of

Table 2 The variables considered during multivariate data analysis

\begin{tabular}{lll}
\hline Variable & Units & Remarks \\
\hline Limit of detection & $\mathrm{ng} \mathrm{g}^{-1}$ & - \\
$\begin{array}{l}\text { Precision } \\
\text { Recovery }\end{array} \%$ & - \\
Amount of sample & $\mathrm{g}$ & - \\
Number of other analytes determined & Unitless & The sample mass subjected to the analysis \\
Amount of organic solvent & $\mathrm{mL}$ & Number of analytes, other than benzo[ $a$ ]pyrene, determined in a single analytical run \\
Amount of organic solvent $\times$ hazard & & The total amount of all organic solvents used in the analytical protocol \\
& & The total amount of each organic solvent used in the analytical protocol multiplied \\
& & by its hazard. In the case of a warning pictogram, the multiplier is 1; in the case \\
Amount of solid waste & $\mathrm{g}$ & The total mass of all waste generated during analysis with the analytical protocol \\
Time & $\mathrm{h}$ & Estimated total time to analyze the sample \\
NEMI score & Unitless (range 0-4) & NEMI score calculated for each analytical method \\
Eco-Scale score & Unitless (range 0-100) & Eco-Scale score calculated for each analytical method \\
\hline
\end{tabular}

NEMI National Environmental Methods Index 
objects (fewer than 25) is an exact method for calculating average ranks available [58]. In the present study, averaged ranks of analytical procedures were calculated by a linear extension set obtained by the Bubley-Dyer algorithm [59].

All calculations concerning the HDT were performed with PyHasse [60] which is available on request from the developer, R. Bruggemann (e-mail: brg_home@web.de).

\section{Results and discussion}

To obtain general information about the dataset structure, PCA was performed; the results are presented in Fig. 1. The LOD, recovery, and precision were not reported in some of source articles listed in Table 1; therefore these variables were not included in the initial assessment.

The results show that the most of the analytical procedures form one group but there are some outliers. For example procedures 2,17 , and 26 , are definitely outliers; they might be potentially performing both much better or much worse according to the variables presented.

The other interesting information is the correlation among the variables. The assessment results obtained by means of NEMI and Eco-Scale are well correlated with each other and they correlate well with the amount of sample. Similarly, the amount of solvent and the hazard-weighted amount of solvent are well correlated, which seems to be obvious. The third group of variables is formed by the analysis time, the amount of solid waste generated during analysis, and the number of other analytes that are determined during a single analysis. This can be interpreted in a way that multianalyte procedures require more time to perform quantitative analyte extraction, which also requires more solid reagent inputs (without recalculation to obtain "per analyte" inputs). All these findings are in agreement with the results of previous studies with the selforganizing maps approach for aldrin determination [61] and benzene and phenol determination [62] in water samples.

The assessment with the HDT was performed for a limited dataset because of low data availability. Some of the variable values (especially recoveries) were not reported in the source articles. Ranking with the HDT for the environmental variables included is shown in Fig. 2.

The results show that procedures 7 and 11 (see Table 1) are ranked as the most environmentally friendly. Both of these methods are characterized by low consumption of solvents which are of low toxicity, require a low sample mass, and produce no waste. The methods present in the maximal objects layer can be treated as the most environmentally

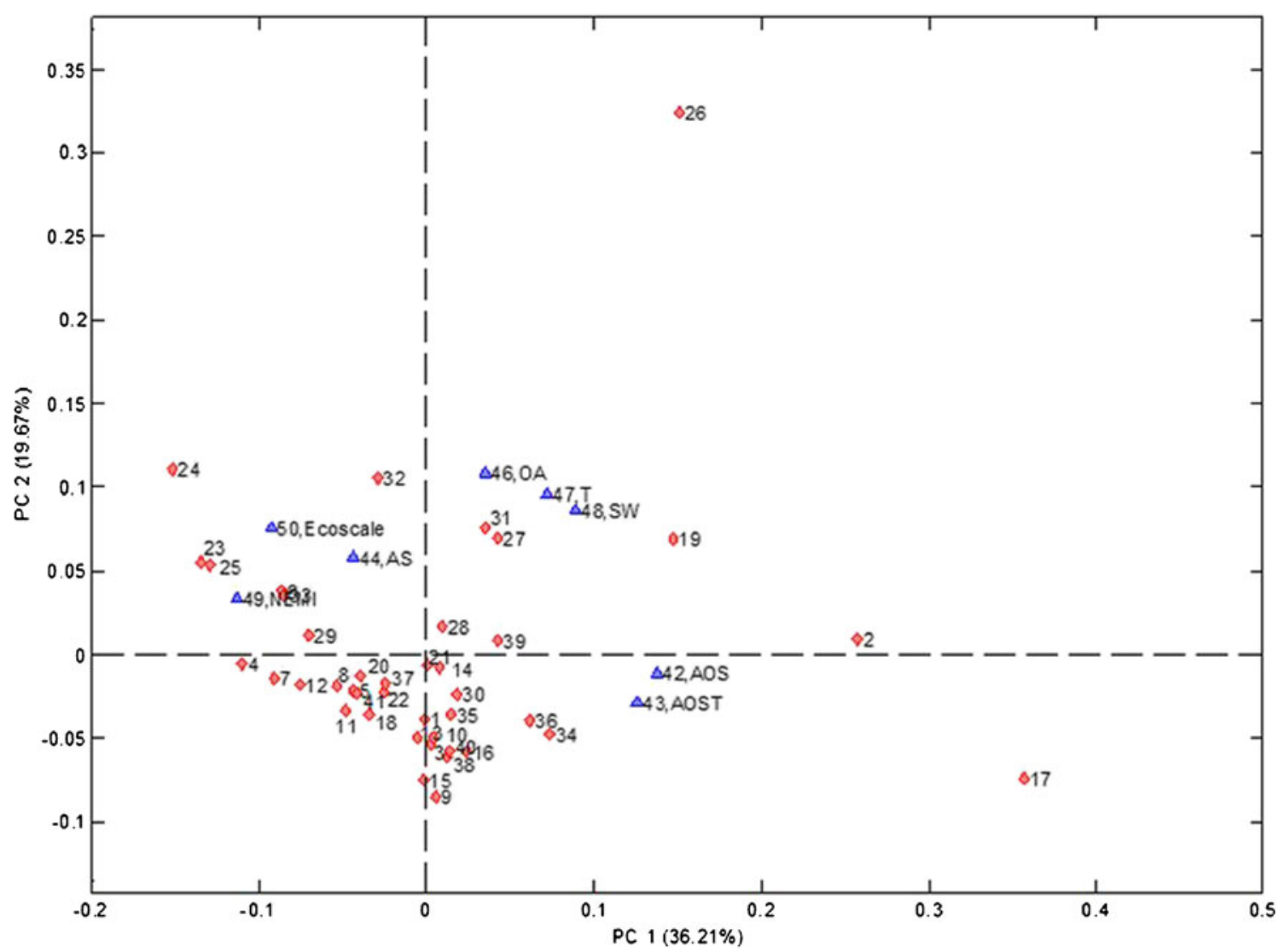

Fig. 1 The results of principal component $(P C)$ analysis for the "environmental" variables from Table 2. AOS amount of organic solvent, AOST amount of organic solvent $\times$ hazard, $A S$ amount of sample, NEMI National Environmental Methods Index, $O A$ number of other analytes determined, $S W$ amount of solid waste, $T$ time 
Fig. 2 Hasse diagram obtained after analysis with the "environmental" variables described in Table 2

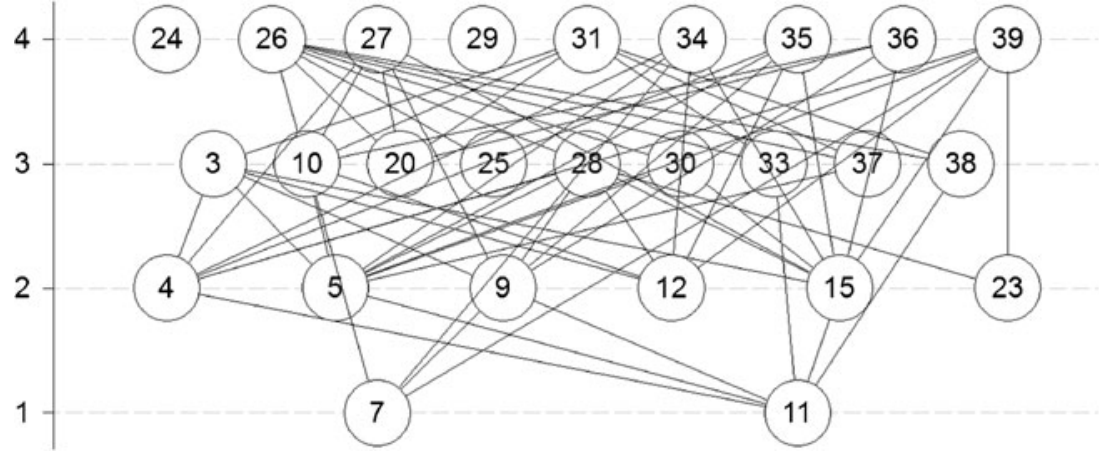

problematic. They are procedures based on pressurized liquid extraction and ultrasonic solid-liquid extraction. This suggests that procedures based on ultrasonic solid-liquid extraction and pressurized liquid extraction are less green than procedures based on microwave-assisted extraction, which are present in the second and third layers of the Hasse diagram. There are also two procedures that are isolated objects. Procedures 24 and 29 (see Table 1) are incomparable with the other procedures. This is because they are characterized by very low values of certain variables and high values of other variables. In this case both procedures consume no or virtually no solvents and produce no waste. On the other hand, the analyses with these procedures are time consuming and require considerable amounts of samples. Both procedures cannot be disregarded as green alternatives and should be treated as a separate group in further considerations.

To check the assessment with the HDT, the results were compared with the results of NEMI and Eco-Scale assessment, which are established assessment procedures and might be treated as reference assessment methods. Comparison with the NEMI assessment (data not shown) results does not show any dependence as most of the methods are scored with two green fields ( $73 \%$ of the methods). The HDT as a green analytical chemistry metrics assessment technique (with well-selected variables as input data) has better resolution power than NEMI labeling. Comparison of the HDT results with the Eco-Scale assessment scores is more informative. The mean Eco-Scale scores for diagram levels 1, 2, 3, and 4 are $83.5,72.5,71.3$ and 67.4 respectively. The mean EcoScale score for two incomparable procedures is 89.5 , which confirms the fact that they also should be treated as green analytical procedures.

Figure 3 presents the HDT assessment results with metrology-related variables as input data. The most beneficial procedures are located at the lowest Hasse diagram level as these procedures are characterized by lower LODs and precision, and recoveries closer to $100 \%$. The best procedures in terms of analytical performance are those based on ultrasound-assisted solvent extraction, followed by gas chromatography-mass spectrometry. On the second level of the Hasse diagram there are mainly methods based on gas chromatography-mass spectrometry based mainly with pressurized liquid extraction as the sample preparation technique. The three objects that are incomparable are objects 4, 11, and 26 (see Table 1). They are characterized by a very low LOD and bad precision or vice versa.

Linear extension analysis of the metrological and environmental performance of analytical procedures for benzo $[a]$ pyrene determination can be very interesting. Figure 4 presents a graph with calculated average ranks according to metrological and environmental variables. The most important message conveyed by Fig. 4 is that there is no procedure that has both good metrological and good environmental performance. A group of procedures (in the green frame in Fig. 4) meet the green analytical chemistry criteria and another group o procedures (in the blue frame in Fig. 4) have good analytical
Fig. 3 Hasse diagram obtained after analysis with the "metrological" variables described in Table 2

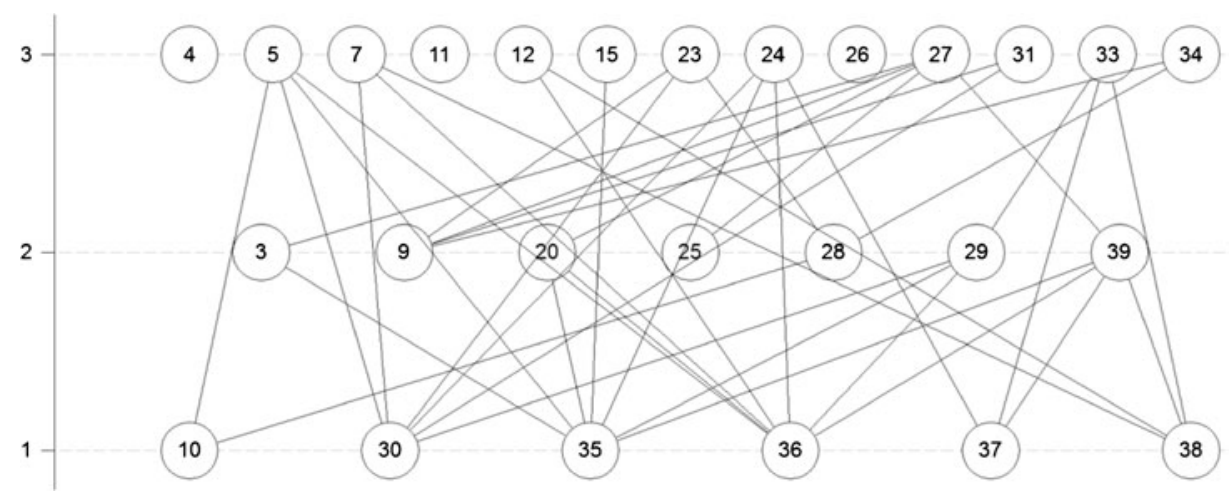


Fig. 4 A bivariate plot of the averaged rank of analytical procedures based on metrological (performance) and environmental (greenness) variables

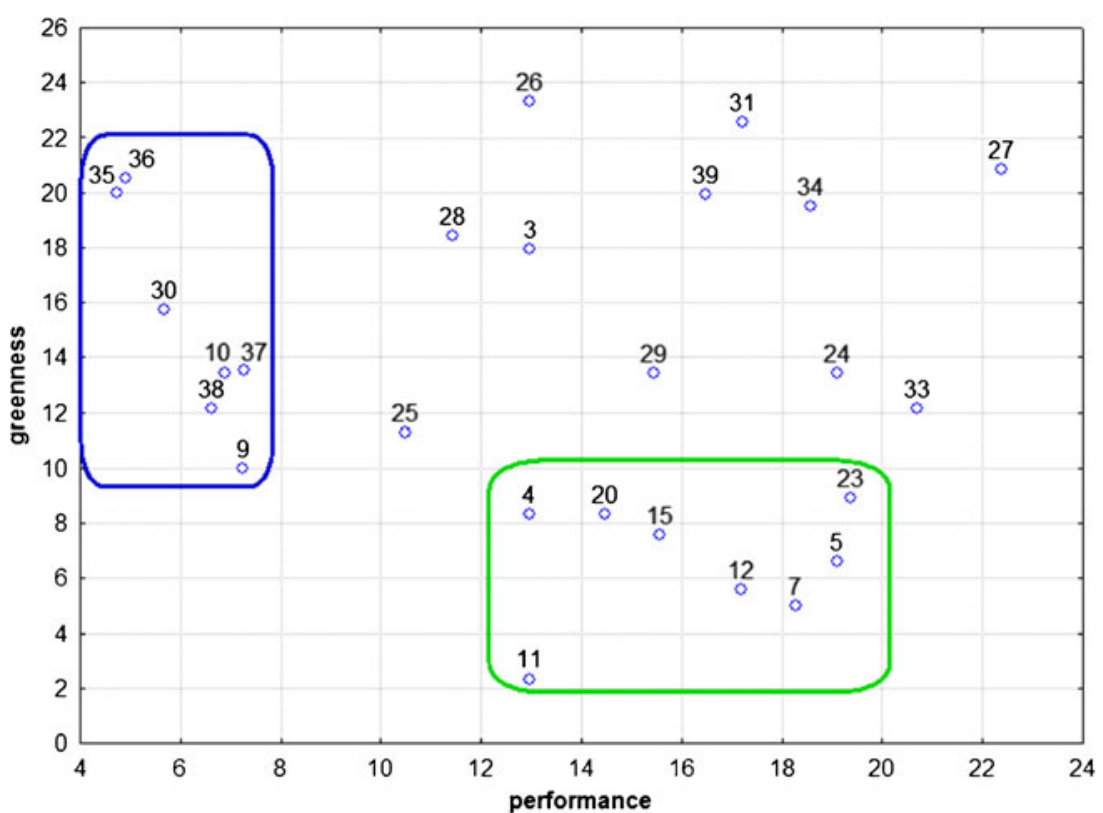

performance. It is hard to choose the procedure that meets both criteria; certain trade-offs are needed. From Fig. 4 it can be easily seen that the procedures in the lower-left part of the diagram $(4,9,11,25$ and 38$)$ have best the compromise of analytical and environmental performances. It is very easy to eliminate the procedures that are characterized by low overall performance - they are located in the upper-right part of the diagram, such as $27,31,34$, and 39 . It can be concluded that depending on the requirements for the particular monitoring study (LOD, precision, etc.), the proper analytical procedure in the lower-left part of the graph can be chosen. It cannot be expected that the HDT will afford a "final decision" about the greenness of a large number of analytical procedures. By the present study we offer a new opportunity for the ranking $\mathrm{O}$ analytical procedures using the most specific variables related to the assessment of the greenness summarized as "performance" and "greenness" parameters. As seen in Fig. 4, quite good assessment is achieved, allowing the ranking of analytical procedures. In our opinion the results sufficiently reflect the goals of the study.

\section{Conclusions}

The HDT can be used as a convenient tool to compare the performance of a set of analytical procedures. The HDT allowed us to choose the best analytical procedures for benzo $[a]$ pyrene determination in sediment samples according to metrological parameters; they were mainly procedures based on ultrasound-assisted extraction. Introduction of variables based on environmental impact as input data for the HDT allowed us to rank the procedures according to their green character. Generally, the procedures based on microwave-assisted extraction were greener, although it is hard to make authoritative generalizations. The HDT was found to be a reliable green analytical chemistry assessment tool. The assessment results are in good agreement with the Eco-Scale assessment results. Another conclusion is that there are no procedures for benzo $[a]$ pyrene determination in sediment samples that are characterized by both good analytical and good environmental performance.

\section{Compliance with ethical standards}

Conflict of interest The authors declare that they have no conflict of interest.

Open Access This article is distributed under the terms of the Creative Commons Attribution 4.0 International License (http:// creativecommons.org/licenses/by/4.0/), which permits unrestricted use, distribution, and reproduction in any medium, provided you give appropriate credit to the original author(s) and the source, provide a link to the Creative Commons license, and indicate if changes were made.

\section{References}

1. Xu P, Ma W, Han H, Jia S, Baolin, Hou. Quantitative structurebiodegradability relationships for biokinetic parameter of polycyclic aromatic hydrocarbons. J Environ Sci. 2015;30:180-5.

2. Rubio-Clemente A, Torres-Palma RA, Penuela GA. Removal of polycyclic aromatic hydrocarbons in aqueous environment by chemical treatments: a review. Sci Total Environ. 2014;478:201-25.

3. US Environmental Protection Agency. Guidelines establishing test procedures for the analysis of pollutants, proposed regulations. Federal Register, vol. 49, no. 209, USEPA, Washington.

4. Jarvis IWH, Dreji K, Masttsson A, Jernstrom B, Stenius U. Interactions between polycyclic aromatic hydrocarbons in complex 
mixtures and implications for cancer risk assessment. Toxicology. 2014;321:27-39.

5. Ba Q, Li J, Huang C, Li J, Chu R, Wu Y, et al. Topological, functional, and dynamic properties of the protein interaction networks rewired by benzo(a)pyrene. Toxicol Appl Pharmacol. 2015;283: 83-91.

6. Liu Y, Wang S, Lohmann R, Yu N, Zhang C, Gao Y. Source apportionment of gaseous and particulate PAHs from traffic emission using tunnel measurements in Shanghai China. Atmos Environ. 2015;107:129-36.

7. Micić V, Kruge MA, Köster J, Hofmann T. Natural, anthropogenic and fossil organic matter in river sediments and suspended particulate matter: a multi-molecular marker approach. Sci Total Environ. 2011;409:905-19.

8. Huang W, Wang Z, Yan W. Distribution and sources of polycyclic aromatic hydrocarbons (PAHs) in sediments from Zhanjiang Bay and Leizhou Bay, South China. Mar Pollut Bull. 2012;64:1962-9.

9. Leite NF, Peralta-Zamora P, Grassi MT. Multifactorial optimization approach for the determination for the determination of polycyclic aromatic hydrocarbons in river sediments by gas chromatographyquadrupole ion trap selected ion storage mass spectrometry. $\mathrm{J}$ Chromatogr A. 2008;1192:273-81.

10. Planas C, Puig A, Rivera J, Caixach J. Analysis of alkyl and 2-6ringed polycyclic aromatic hydrocarbons by isotope dilution gas chromatography/mass spectrometry. Quality assurance and determination in Spanish river sediments. J Chromatogr A. 2006;1113: 220-30.

11. Pino V, Anderson JL, Ayala JH, Gonzalez V, Afonso AM. The ionic liquid 1-hexadecyl 3-methylimidazolium bromide as novel extracting system for polycyclic aromatic hydrocarbons contained in sediments using focused microwave-assisted extraction. J Chromatogr A. 2008;1182:145-52.

12. US EPA method 3540C - Soxhlet extraction $1996 \mathrm{http}: / / w w w . e p a$. gov/osw/hazard/testmethods/sw846/pdfs/3540c.pdf.

13. Kimbrough KL, Lauenstein GG, Johnson WE. Organic contaminant analytical methods of the National Status and Trends Program: update 2000-2006. NOAA technical memorandum NOS NCCOS 2006;30:137.

14. Spietelun A, Marcinkowski $Ł$, de la Guardia M, Namieśnik J. Green aspects developments and perspectives of liquid phase microextraction techniques. Talanta. 2014;119:34-45.

15. Pena-Pereira F, Lavilla I, Bendicho C. Liquid-phase microextraction techniques within the framework of green chemistry. Trends Anal Chem. 2010;29:617-28.

16. Gałuszka A, Migaszewski Z, Namieśnik J. The 12 principles of green analytical chemistry and the SIGNIFICANCE mnemonic of green analytical practices. Trends Anal Chem. 2013;50:78-84.

17. Farre M, Perez S, Goncalves C, Alpendurada MF, Barcelo D. Green analytical chemistry in the determination of organic pollutants in the aquatic environment. Trends Anal Chem. 2010;29:1347-62.

18. Kaljurand M, Koel M. Recent advancements on greening analytical separation. Crit Rev Anal Chem. 2011;4:12-20.

19. Keith LH, Gron LU, Young JL. Green analytical methodologies. Chem Rev. 2007;107:2695-708.

20. Gałuszka A, Konieczka P, Migaszewski ZM, Namieśnik J. Analytical Eco-Scale for assessing the greenness of analytical procedures. Trends Anal Chem. 2012;37:61-72.

21. Filipkowska A, Lubecki L, Kowalewska G. Polycyclic aromatic hydrocarbon analysis in different matrices of the marine environment. Anal Chim Acta. 2005;547:243-54

22. Williamson KS, Petty JD, Huckins JN, Lebo JA, Kaiser EM. HPLC-PFD determination of priority pollutant PAHs in water sediment and semipermeable membrane devices. Chemosphere. 2003;49:703-15.

23. Peng X, Yan G, Li X, Guo X, Zhou X, Wang Y. Optimization of ultrasonic extraction and clean-up protocol for the determination of polycyclic aromatic hydrocarbons in marine sediments by highperformance liquid chromatography coupled with fluorescence detection. J Ocean Univ China. 2012;11:331-8.

24. Pino V, Ayala JH, Afonso AM, Gonzalez V. Ultrasonic micellar extraction of polycyclic aromatic hydrocarbons from marine sediments. Talanta. 2001;54:15-23

25. Leng G, Lui G, Chen Y, Yin H, Dan D. Vortex-assisted extraction combined with dispersive liquid-liquid microextraction for the determination of polycyclic aromatic hydrocarbons in sediment by high performance liquid chromatography. J Sep Sci. 2012;35: 2796-804.

26. Moliner-Martinez Y, Gonzalez-Fuenzalida RA, Herraez-Hernandez R, Campins-Falco P, Verdu-Andres J. Cleaning sorbents used in matrix solid-phase dispersion with sonification: application to the estimation of polycyclic aromatic hydrocarbons at $\mathrm{ng} / \mathrm{g}$ levels in marine sediments. J Chromatogr A. 2012;1263:43-50.

27. Capelo JL, Galesio MM, Felisberto GM, Vaz C, Pessoa JC. Microfocused ultrasonic solid-liquid extraction (FUSLE) combined with HPLC and fluorescence detection for PAHs determination in sediments: optimization and linking with the analytical minimalism concept. Talanta. 2005;66:1272-80.

28. Shampsipur M, Hassan J. A novel miniaturized homogenous liquid-liquid solvent extraction-high performance liquid chromatographic-fluorescence method for determination of ultra traces of polycyclic aromatic hydrocarbons in sediments samples. J Chromatogr A. 2010;1217:4877-82.

29. Gonazlo-Pinuela C, Alonso-Salces RM, Andres A, Ortiz I, Viguri JR. Validated analytical strategy for the determination of polycyclic aromatic compounds in marine sediments by liquid chromatography coupled with diode-array detection and mass spectrometry. J Chromatogr A. 2006;1129:189-200.

30. Delgado B, Pino V, Anderson JL, Ayala JH, Afonso AM, Gonzalez V. An in-situ extraction-preconcentration method using ionic liquid-based surfactants for the determination of organic contaminants contained in marine sediments. Talanta. 2012;99:972-83.

31. Denis EH, Toney JL, Tarozo R, Anderson RS, Roach LD, Huang Y. Polycyclic aromatic hydrocarbons (PAHs) in lake sediments record historic fire events: validations using HPLC-fluorescence detection. Org Geochem. 2012;45:7-17.

32. Thompson S, Budzinski H, LeMenach K, Letellier M, Garrigues P. Maulti-residue analysis of polycyclic aromatic hydrocarbons, polychlorobiphenyls, and organochlorine pesticides in marine sediments. Anal Bioanal Chem. 2002;372:196-204.

33. Pena-Abaurrea M, Ye F, Blasco J, Ramos L. Evaluation of comprehensive two-dimensional gas chromatography-time-of-flight-mass spectrometry for the analytes of polycyclic hydrocarbons in sediments. J Chromatogr A. 2012;1256:222-31.

34. Shu YY, Lao RC, Chiu CH, Turle R. Analysis of polycyclic aromatic hydrocarbons in sediment reference materials by microwaveassisted extraction. Chemosphere. 2000;41:1709-16.

35. Budziński H, Letellier M, Garrigues P, Le Menach K. Optimisation of the microwave-assisted extraction in open cell of polycyclic aromatic hydrocarbons from soils and sediments. Study of moisture effect. J Chromatogr A. 1999;837:187-200.

36. Notar M, Leskovsek H. Determination of polycyclic aromatic hydrocarbons in marine sediments using a new ASE-SFE extraction technique. Fresenius J Anal Chem. 2000;366:846-50.

37. Errekatxo A, Prieto A, Zuloaga O, Usobiaga A, Etxebarria N, Fernandez LA. Simultaneous extraction of several persistent organic pollutants in sediments using focused ultrasonic solid-liquid extraction. Anal Bioanal Chem. 2008;392:1471-8.

38. Itoh N, Numata $\mathrm{M}$, Yarita T. Alkaline extraction in combination with microwave-assisted extraction followed by solid-phase extraction treatment for polycyclic aromatic hydrocarbons in sediment sample. Anal Chim Acta. 2008;615:47-53. 
39. Herbert P, Silva AL, Joao MJ, Santos L, Alves A. Determination of semi-volatile priority pollutants in landfill leachates and sediments using microwave-assisted headspace solid-phase microextraction. Anal Bioanal Chem. 2006;386:324-31.

40. Pino V, Ayala JH, Afonso AM, Gonzalez V. Micellar microwaveassisted extraction combined with solid-phase microextraction for the determination of polycyclic aromatic hydrocarbons in certified marine sediment. Anal Chim Acta. 2003;477:81-91.

41. Fernandez-Gonalez V, Concha-Grana E, Muniategui-Lorenzo S, Lopez-Mahia P, Prada-Rodriquez D. Pressurized hot water extraction coupled to solid-phase microextraction-gas chromatographymass spectrometry for analysis of polycyclic aromatic hydrocarbons in sediments. J Chromatogr A. 2008;1196-1197:65-72.

42. Camino-Sanchez FJ, Zafra-Gomez A, Perez-Trujillo JP, CondeGonzalez JE, Mrques JC, Vilchez JL. Validation of a GC-MS/MS method for simultaneous determination of 86 persistent organic pollutants in marine sediments by pressurized liquid extraction followed by stir bar sorptive extraction. Chemosphere. 2011;84:869-81.

43. Burkhardt MR, Re R, Vello RC, Smith SG, Zaugg SD. Pressurized liquid extraction using water/isopropanol coupled with solid-phase extraction cleanup for industrial and anthropogenic waste-indicator compounds in sediment. Anal Chim Acta. 2005;534:89-100.

44. Choi M, Kim Y-J, Lee IS, Choi H-G. Development of a one-step integrated pressurized liquid extraction and cleanup method for determining polycyclic aromatic hydrocarbons in marine sediments. J Chromatogr A. 2014;1340:8-14.

45. Ramos L, Vreuls JJ, Brinkman UAT. Minaturised pressurised liquid extraction of polycyclic of aromatic hydrocarbons from soil and sediment with subsequent large-volume injection-gas chromatography. J Chromatogr A. 2000;891:275-86.

46. Fernandez-Gonalez V, Concha-Grana E, Muniategui-Lorenzo S, Lopez-Mahia P, Prada-Rodriquez D. A multivariate study of the programmed temperature vaporization injection-gas chromatography-mass spectrometric determination of polycyclic aromatic hydrocarbons. Application to marine sediments analysis. Talanta. 2008;74:1096-103.

47. Wolska L. Miniaturised analytical procedure of determining polycyclic aromatic hydrocarbons and polychlorinated biphenyls in bottom sediments. J Chromatogr A. 2002;959:173-80.

48. Shang D. Rapid and sensitive method for the determination of polycyclic aromatic hydrocarbons in soils using pseudo multiple reaction monitoring gas chromatography/tandem mass spectrometry. J Chromatogr A. 2014;1334:113-25.

49. Martinez E, Gros M, Lacorte S, Barcelo D. Simplified procedures for the analysis of polycyclic aromatic hydrocarbons in water, sediments and mussels. J Chromatogr A. 2004;1047:181-8.
50. Banjoo DR, Nelson PK. Improved ultrasonic extraction procedure for the determination of polycyclic aromatic hydrocarbons in sediments. J Chromatogr A. 2005;1066:9-18.

51. Yamaguchi C, Lee W-Y. A cost effective, sensitive, and environmentally friendly sample preparation method for determination of polycyclic aromatic hydrocarbons in solid samples. J Chromatogr A. 2010;1217:6816-23.

52. Sanchez-Avila J, Fernandez-Sanjuan M, Vincente J, Lacorte S. Development of a multi-residue method for the determination of organic micropollutants in water, sediment and mussels using gas chromatography-tandem mass spectrometry. J Chromatogr A. 2011;1218:6799-811.

53. Ericsson M, Colmsjo A. Dynamic microwave-assisted extraction coupled on-line with solid-phase extraction: determination of polycyclic aromatic hydrocarbons in sediment and soil. J Chromatogr A. 2002;964:11-20.

54. Bruggemann R, Halfon E, Welzl G, Voigt K, Steinberg C. Applying the concept of partially ordered sets on the ranking of near-shore sediments by a battery of tests. J Chem Inf Comput Sci. 2001;41: 918-25.

55. Bruggemann R, Voigt K. Basic principles of Hasse diagram technique in chemistry. Comb Chem High Throughput Screen. 2008;11:756-69.

56. Voyslavov T, Tsakovski S, Simeonov V. Hasse diagram technique as a tool for water quality assessment. Anal Chim Acta. 2013;770: 29-35.

57. Carlsen L, Bruggemann R, Kenessova O, Erzhigitov E. Evaluation of analytical performance based on partial order methodology. Talanta. 2015;132:285-93.

58. De Loof K, De Meyer H, De Baets B. Exploiting the lattice of ideals representation of a pose. Fundam Inf. 2006;71:309-21.

59. Bubley R, Dyer M. Faster random generation of linear extensions. Discret Math. 1999;201:81-8.

60. Bruggemann R, Carlsen L, Voigt Wieland KR. In: Bruggemann R, Carlsen L, Wittmann J, editors. Multiindicator systems and modelling in partial order. New York: Springer; 2014. p. 389-423.

61. Tobiszewski M, Tsakovski S, Simeonov V, Namieśnik J. Application of multivariate statistics in assessment of green analytical chemistry parameters of analytical methodologies. Green Chem. 2013;15:1615-23.

62. Tobiszewski M, Tsakovski S, Simeonov V, Namieśnik J. Multivariate statistical comparison of analytical procedures for benzene and phenol determination with respect to their environmental impact. Talanta. 2014;130:449-55. 